\title{
The vertical structure of the magnetic field of the CP star $\alpha^{2} \mathrm{CVn}$
}

\author{
I. Romanyuk and D. Kudryavtsev \\ Special Astrophysical Observatory of the RAS, Nizhny Arkhyz, Russia \\ email: roman@sao.ru, dkudr@sao.ru
}

\begin{abstract}
We observed the magnetic field of the CP star $\alpha^{2} \mathrm{CVn}$ with the 6-m telescope and the echelle-spectrometer (spectral resolution $R=50000$, spectral range $\lambda \lambda 3400-4100$ ) using a CCD device and Zeeman analyzer. The longitudinal magnetic field $B_{\mathrm{e}}$ measured using lines with wavelengths shorter than the Balmer jump ( $\lambda 3646)$ are systematically $25 \%$ smaller at all rotational phases compared with measurements made in the region $\lambda>3646$. Because in general lines with $\lambda>3646$ form at deeper levels than lines with $\lambda<3646$ we get additional evidence for the increase of $B_{\mathrm{e}}$ with depth in the atmosphere of $\alpha^{2} \mathrm{CVn}$.
\end{abstract}

Keywords. Stars: chemically peculiar, stars: magnetic fields, stars: atmosphere, stars: individual $\left(\alpha^{2} \mathrm{CVn}\right)$

\section{Introduction}

Preston (1969) was the first to propose a search for the radial (vertical) gradient of the magnetic fields of CP stars using lines formed at different depths of their atmospheres. He used lines with different intensities and found the magnetic field for the CP star 53 Cam to increase with decreasing line intensity in its spectrum. Romanyuk (1980) confirmed Preston's result and found additional evidence for the existence of a radial gradient of the magnetic field in the CP stars 53 Cam (HD 65339) and $\alpha^{2}$ CVn (HD 112413).

Wolff (1978) was the first to measure Zeeman spectra for 3 stars obtained with the wavelengths shorter than the Balmer jump ( $\lambda 3646)$. Lines with $\lambda<3646$ are mostly formed in higher layers of the atmosphere than lines in most commonly used spectral range $\lambda>3646$. She found for $\alpha^{2} \mathrm{CVn}$ a small decrease of the longitudinal magnetic field strength in the higher layers of atmosphere. For another magnetic star $\beta \mathrm{CrB}$ (HD 137909), the magnetic field strength is the same below and above the Balmer jump.

We improved this technique and made a series of observation in the 1980's using the 6-m telescope and photographic plates with an achromatic Zeeman analyzer in spectral range $\lambda \lambda$ 3000-7000 (Romanyuk 1984, 1986, 1991). This work showed that the longitudinal component of magnetic field of $\alpha^{2} \mathrm{CVn}$ has a smaller value below the Balmer jump and the amplitude of variations agree with Wolff's result, while for $\beta \mathrm{CrB}$ such an effect is not observed. As the differences are very small, their reliability is open to discussion due to a possible distortion caused by the non-linearity of photographic emulsion sensitivities. For this reason, 15 years later we began a new series of observations using CCD devices.

\section{Observations and data reduction}

Our program of observations with the 6 -m telescope includes several bright magnetic $\mathrm{CP}$ stars. To date, a sufficient number of values have been obtained only for $\alpha^{2} \mathrm{CVn}$, the brightest magnetic star. The longitudinal component of its magnetic field was measured many times and published in dozens of papers. The results show that the longitudinal 
Table 1. Magnetic field in $\alpha^{2}$ CVn before and after the Balmer jump

\begin{tabular}{lrlrlr}
\hline JD 245000+ phase $\begin{array}{l}B_{\mathrm{e}} \pm \sigma(\mathrm{G}) \\
\lambda<3646\end{array}$ & $\mathrm{n} \begin{array}{l}B_{\mathrm{e}} \pm \sigma(\mathrm{G}) \\
\lambda>3646\end{array}$ & $\mathrm{n}$ \\
\hline 1385.258 & 0.167 & $-880 \pm 310$ & 25 & $-1130 \pm 110$ & 59 \\
1591.415 & 0.860 & $-800 \pm 350$ & 110 & $-1000 \pm 140$ & 68 \\
1592.404 & 0.042 & $-980 \pm 270$ & 74 & $-1130 \pm 110$ & 78 \\
1593.386 & 0.219 & $-410 \pm 480$ & 76 & $-1390 \pm 150$ & 74 \\
2594.583 & 0.275 & $-1020 \pm 330$ & 71 & $-1420 \pm 100$ & 327 \\
2595.600 & 0.461 & $+1660 \pm 260$ & 47 & $+1970 \pm 110$ & 290 \\
2596.588 & 0.641 & $-500 \pm 310$ & 37 & $-1140 \pm 100$ & 283 \\
\hline
\end{tabular}

component of the magnetic field Be measured by lines of metals in the usual spectral range changes from -1600 to $+1800 \mathrm{G}$. The $B_{\mathrm{e}}$ curve is anharmonic with the positive extremum narrower and sharper than the negative one. Measurements made using a Balmer line magnetometer and hydrogen lines (Borra \& Landstreet 1977) show a practically sinusoidal curve $B_{\mathrm{e}}$ with a slightly lower amplitude of variation. The period of all variations (photometric, spectral and magnetic) is that of rotation and can be described by the elements of Farnsworth (1932):

$$
J D=2419869.72+5.46939 E \text { (days). }
$$

Our new observations were conducted with the echelle-spectrometer NES and a CCD detector with $2000 \times 2000$ elements in 2000-2003. The procedures are described and the details of observation are given in Panchuk et al. (2000) and Romanyuk et al. (2004). We obtained Zeeman spectra in the wide region $\lambda \lambda 3400-4100$ with a resolution of 50000 . The $S / N$ ratio at $\lambda 3600$ was 50 , while at $\lambda 3900$ it was about 200 ; the differences are due to a drop of CCD sensitivity and Zeeman analyzer transparency in the optical ultraviolet. This leads to a lower measurement accuracy in the region shortward of the Balmer jump.

The reduction of observations was made in ESO MIDAS using the programs for Zeeman spectra reduction (Kudryavtsev 2000).

\section{Discussion of results}

The results of our observations are presented in Table 1 and Figure 1. Table 1 gives for each observation the Julian date of observation, the rotational phase, the longitudinal magnetic field $B_{\mathrm{e}}$, and the mean-square error $\sigma$ in the spectral range below and above the Balmer jump, and $n$ the number of measured lines for each spectrum. They confirm the well-known fact that measurements from metallic lines in the usual spectral region (asterisks in Figure 1) produce an anharmonic curve for the longitudinal magnetic field $B_{\mathrm{e}}$ with a larger amplitude of variability than the field curve obtained from hydrogen lines (filled dots).

The measurements in the region shorter than $\lambda 3646$ (open dots) show that the field strength in the upper atmospheric layer is much closer to that derived from hydrogen lines in both the amplitude of variability and the shape of the curve.

At the phases of extrema of $B_{\mathrm{e}}$, the field magnitude from lines formed in deeper layers of the atmosphere is by about $25 \%$ larger than in upper layers. This confirms our previous result obtained from photographic plates. We think that the differences between the photographic and photoelectric curves of the longitudinal field are associated not only with methodological causes (Borra 1974). First, hydrogen in contrast to metals, is uniformly distributed over the surface of $\alpha^{2} \mathrm{CVn}$. Second, the measurements with 


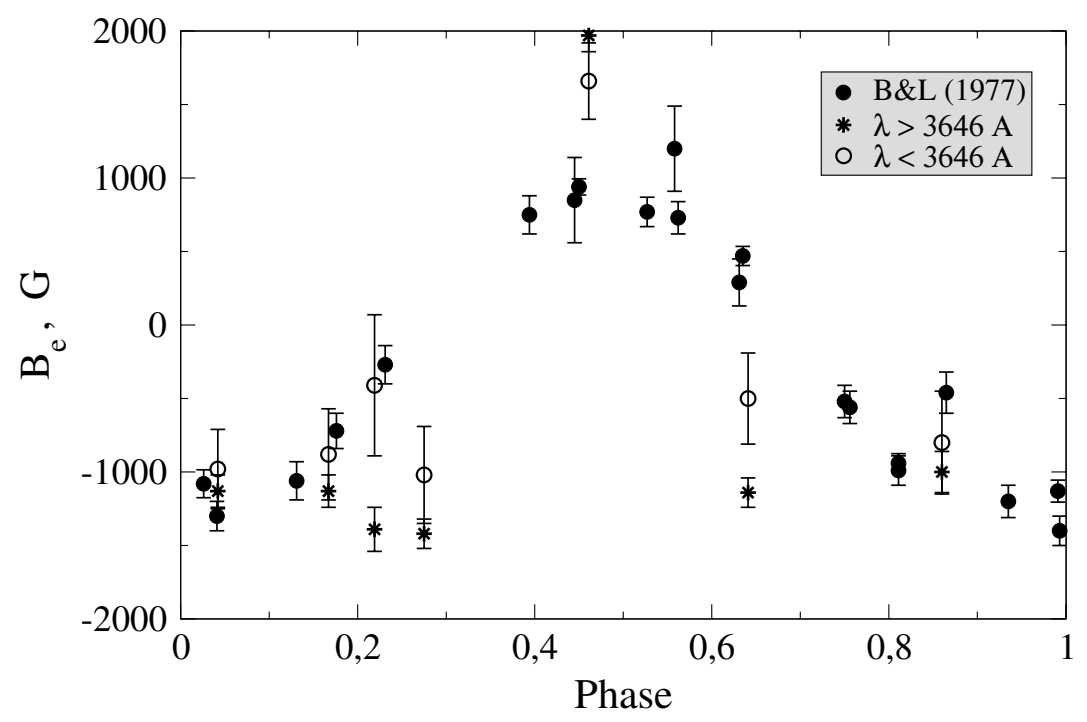

Figure 1. The magnetic field of $\alpha^{2} \mathrm{CVn}$. Filled circles - measurements by Borra \& Landstreet (1977) with Balmer-line magnetometer; asterisks — our CCD measurements using lines of metals in the spectral region $\lambda>3646$; open circles — our CCD measurements using lines of metals in the spectral region with $\lambda<3646$.

photoelectric magnetometer were performed in a hydrogen line wing, but close to its core, where the signal of circular polarization reaches its maximum value. Hydrogen line cores form in the higher layers of the atmosphere than their wings. Thus differences in hydrogen and metallic curves of $B_{\mathrm{e}}$ may also be due to changes in the field with depth.

The lines in the region shortward of the Balmer jump form at approximately the same depth as the cores of hydrogen lines, therefore the magnetic field measured from this region resembles the field measured from hydrogen.

Thus, we have obtained additional information about the strength and the complications of the structure of the magnetic field of $\alpha^{2} \mathrm{CVn}$ with depth in its atmosphere. Future observations are necessary to select stars whose hydrogen and metallic curves are greatly different.

\section{References}

Borra, E.F. 1974, AJ 188, 287

Borra, E.F., Landstreet, J.D. 1977, AJ 212, 141

Farnsworth, G. 1932, AJ 76, 313

Kudryavtsev, D. 2000, in: Yu.V. Glagolevskij \& I.I. Romanyuk (eds.), Magnetic fields of CP and related stars, (Moscow), p. 23

Panchuk, V.E., Romanyuk, I.I., Kudryavtsev, D.O. 2000, in: Yu.V. Glagolevskij \& I.I. Romanyuk (eds.), Magnetic fields of $C P$ and related stars, (Moscow), p. 76

Preston, G. 1969, AJ 157, 247

Romanyuk, I.I 1980, Astrofiz. Issled., (Izv. SAO) 12, 3

Romanyuk, I.I. 1984, Pis'ma v AZh 10, 443

Romanyuk, I.I. 1986, Astrofiz. Issled., (Izv. SAO) 22, 25

Romanyuk, I.I. 1991, Astrofiz. Issled. (Izv. SAO) 33, 53

Romanyuk, I.I., Panchuk, V.E., Piskunov, N.E., Kudryavtsev, D.O. 2004, (in preparation)

Wolff, S.C. 1978, PASP 90, 412 